\title{
Elementary Processes of Response Selection Mediated by Distinct Regions of the Striatum
}

\author{
Verity J. Brown and Trevor W. Robbins \\ Department of Experimental Psychology, University of Cambridge, Cambridge CB2 3EB, United Kingdom
}

The relative contributions of the medial and lateral caudate putamen to performance of a visual reaction time task were compared by examining the effects of unilateral ibotenateinduced lesions to these regions in the rat. Different groups of rats were trained to respond either towards (Discrimination 1) or away from (Discrimination 2) unpredictable, brief visual stimuli presented to either side of the head.

Lateral striatal lesions produced a strong spatial bias towards the side of the lesion but left the latency for the initiation of responses to the visual cues unchanged. By contrast, the medial striatal lesions resulted in a smaller degree of spatial response bias but a significant slowing of initiation latency to the side contralateral to the lesion. These effects were irrespective of the side of the stimulus presentation and thus represent response-related impairments. The lesions were further dissociated in their effects on responding inappropriately to task requirements.

These results demonstrate a double dissociation of behavioral effects of lateral and medial striatal damage that can be used to infer the operation of distinct elementary processes of response output within a single task. They are important not only in demonstrating functional effects of hypothetical segregated parallel corticostriatal loops but also in showing that these loops must interact to produce integrated performance.

Anatomical, biochemical, and behavioral evidence indicates that the striatum is heterogeneous with respect to function (Divac et al., 1967; Dunnett and Iversen, 1980, 1982a, b; Divac, 1983; Graybiel and Ragsdale, 1983; Pisa, 1988). From this evidence, it is clear that the striatum should not be regarded as a functional "center" independent of its anatomically related cortical areas (Divac, 1972; Alexander et al., 1986). For example, in the manifestation of different aspects of contralateral "neglect" such as skilled paw use (Sabol et al., 1985; Pisa, 1988), somatosensory orientation (Dunnett and Iversen, 1982a; Fairley and Marshall, 1986), and recovery after tissue transplantation (Dunnett et al., $1981)$, it is widely agreed that the lateral caudate putamen is particularly significant. Lesions of dorsolateral frontoparietal

\footnotetext{
Received Feb. 13, 1989; revised Apr. 21, 1989; accepted Apr. 24, 1989.

This research was supported by the Wellcome Trust, U.K. V.J.B. is in receipt of a Medical Research Council (U.K.) Studentship. We wish to thank Dr. T. Shallice for helpful discussions and Dr. B. Everitt for comments on the manuscript.

Correspondence should be addressed to Trevor W. Robbins, Department of Experimental Psychology, University of Cambridge, Downing Street, Cambridge CB2 3EB, U.K.

Copyright (C) 1989 Society for Neuroscience $0270-6474 / 89 / 113760-06 \$ 02.00 / 0$
}

cortex also impair forelimb reaching (Castro, 1972), and the projections from this region to the lateral caudate putamen of the rat would suggest that these areas form a "motor" circuit of the type envisaged by Alexander et al. (1986).

The medial portion of the striatum of the rat receives projections from frontocingulate cortex (Nauta and Domesick, 1984), which has also been implicated in behavioral neglect in the rat (Cowey and Bozek, 1974; Barth et al., 1982; Crowne and Pathria, 1982). According to the parallel loop model of Alexander et al. (1986), it might then be expected that lesions of medial striatum would have similar effects, although so far the experimental evidence for this is weak.

If both the lateral and the medial areas of the striatum are implicated in neglect, then their different contributions to the control of behavior, as well as the ways in which they interact as components of separate parallel functional loops, require definition. This problem of defining how these corticostriatal functional loops are both integrated and interact can be best determined by examining performance of different aspects of a single task.

In this experiment, the relative contributions of the medial and lateral caudate putamen were examined in a visual reaction time task developed for the rat (Carli et al., 1985). The design of the task enables the dissociation of sensory and output related factors in neglect and allows an assessment of how behavioral processes subserved by distinct subregions of the striatum may determine different aspects of performance of a single task.

\section{Materials and Methods}

\section{Animals}

The subjects were 24 male Lister hooded rats (Olac, Bicester, UK), weighing 200-250 gm at the beginning of the experiment and maintained at $90 \%$ of their free-feeding weight with $15 \mathrm{gm} / \mathrm{d} / \mathrm{rat}$ of standard laboratory chow, given after the experimental sessions. Water was freely available throughout. The animals were housed in pairs under a natural light-dark cycle.

\section{Surgery}

After training to stable performance, unilateral lesions of the caudate putamen were induced by infusion of the excitotoxin, ibotenic acid. Surgery was performed under Equithesin anesthesia $(3 \mathrm{ml} / \mathrm{kg}$, i.p.) in clean conditions. Animals were placed in a Kopf stereotaxic instrument, and lesions were made at the following placements (Pellegrino et al., 1979): medial, A +2.5, L 1.8, V -4.0; lateral, A +2.5, L 3.5, V -5.5. One microliter of $0.06 \mathrm{M}(9.4 \mu \mathrm{g} / \mu \mathrm{l})$ ibotenic acid (Sigma, UK) in phosphate buffer was infused at a constant rate of $0.5 \mu \mathrm{l} / \mathrm{min}$ via a 30 gauge stainless steel cannula attached to a Harvard infusion pump. The cannula was left in place for 2 min before and after the infusion. Of the animals trained on Discrimination 1 (see below), 7 received medial and 6 , lateral lesions. Of those trained on Discrimination 2, 6 received medial and 5, lateral lesions. 


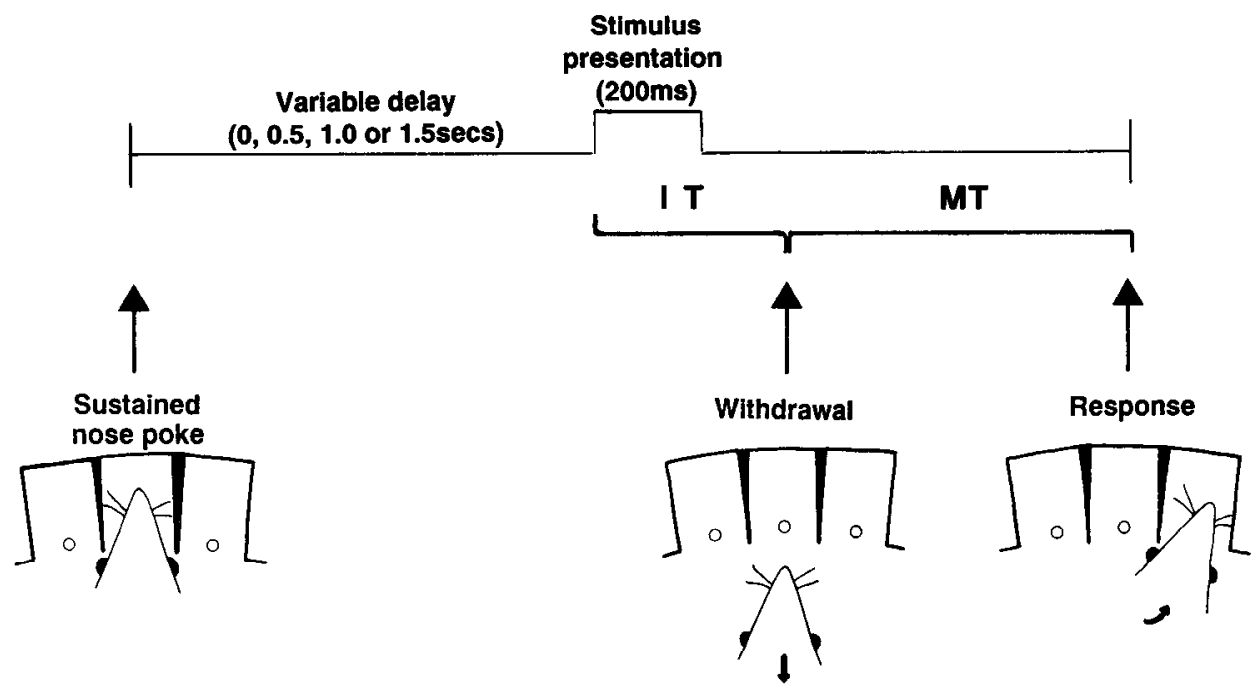

Figure 1. Schematic diagram illustrating the composition of a single trial. A brief visual stimulus is presented in one of the side holes after a variable delay. Initiation time (IT) is the time from the onset of the stimulus to the withdrawal of the head from the central hole, and movement time (MT) is the latency from that point to the response in one of the side holes. In Discrimination 1 , the response is correct if it corresponds to the side of the stimulus presentation. In Discrimination 2, the response is correct if it is made opposite to the side of the stimulus.

\section{Histology}

At the end of the experiment, the animals were deeply anesthetized and then transcardially perfused with $0.9 \%$ saline followed by $10 \%$ formalinsaline. The brains were postfixed for $4 \mathrm{hr}$ and then stored in $30 \%$ sucrose before being sectioned at $60 \mu \mathrm{m}$ on a freezing microtome. Every 3rd and 4th section was mounted and stained with either cresyl violet or for acetylcholinesterase (AChE) by the method of Koelle (1954).

The areas of cell loss and gliosis revealed by cresyl violet staining and decreased striatal AChE staining were mapped onto standardized sections of the brain. The areas of the lesions were calculated and multiplied by the distance between sections to give an estimate of total lesion volume.

\section{Apparatus}

The apparatus was as previously described (see figure 1 of Carli et al., 1983; Brown and Robbins, 1989). Three operant chambers were used, each with a horizontal array of $2.5 \mathrm{~cm}^{2}$ holes set into the rear wall. Three adjacent holes in the array were open to the animal, with a vertically dirccted photoccll beam across the front of each hole which registered responses. Illumination of a $2.8 \mathrm{~W}$ bulb situated at the rear of each hole was used as the visual stimulus. The boxes were controlled and the data collected online by microcomputers.

\section{Procedure}

Training. Following preliminary training to collect pellets $(45 \mathrm{mg}$ dustless precision pellets, BioServ Inc., $\mathrm{NJ}$ ) from the food magazine, the rats were rewarded on a continuous reinforcement schedule for making nose-poke responses into the lit central hole. When they were responding reliably, the control program was implemented; a correction procedure was used for incorrect trials until stable performance of around $80 \%$ correct was reached. The trial was initiated by the rat by pushing the Plexiglas panel in front of the food magazine. The central hole was illuminated and a sustained nose-poke in the hole extinguished that light and began 1 of 4 intervals (delays) before the presentation of the brief $(200 \mathrm{msec})$ visual stimulus in either one of the side holes. The delays, which were initially very short, were progressively increased over several weeks until the rat was waiting, with its nose in the central hole, for delays of $0,0.5,1.0$, or $1.5 \mathrm{sec}$.

The rat was required to report the location of the light by withdrawing its nose from the central hole and responding in one of the side holes. The composition of a trial is shown in Figure 1. Thirteen rats were trained on Discrimination 1, to move towards the side of the light, while 11 were trained on Discrimination 2, to move away from the stimulus.

Correct responses were rewarded by the delivery of a food pellet. Incorrect responses, or premature withdrawals from the central hole, were punished with a $1 \mathrm{sec}$ period of darkness (Time-out).

Baseline performance. For each of the 2 response holes, there were 10 trials at each of 4 delays ( 80 trials), presented in a pseudo-random

order. To obtain the preoperative baseline values, rats were tested for 5 consecutive days prior to surgery. Postoperative testing commenced after a period of recovery lasting $5 \mathrm{~d}$.

Effects of manipulating the visual stimuli. The rats were tested on probe trials, interspersed throughout a single session, in which both the stimuli were presented simultaneously, in a test analogous to the clinical test for sensory extinction (Benton, 1956). In addition, 2 sessions were given when the stimulus light of one of the response holes was removed. Without a stimulus, the animals nevertheless eventually made a response. The effect of removing one of the stimuli was therefore to lengthen initiation times of responses which would have been cued by the missing stimulus. By comparing performance under these conditions with that in the baseline condition, it may be ascertained whether any deficits seen in baseline performance could be attributed to an inability to detect one of the stimuli.

\section{Measures and statistical analysis}

Latency to initiate correct and incorrect responses (taken as the time from the onset of the stimulus to withdrawal from the central hole) was measured as initiation time (IT). No correction was made for the systematic error inherent in using a photocell detection system, which leads to overestimates of IT proportional to the time it takes for the snout to leave the beam. This will be a function of the distance the head intrudes into the hole and the velocity with which it leaves it, but it seems likely that these errors are constant and small in relation to measured IT. Movement time (MT) was the time to complete the response from the withdrawal from the central hole to the response in the side hole. All response times were measured with centisecond accuracy. Percent correct was recorded, and response bias was expressed as responses (correct and incorrect) made to the ipsilateral side as a percentage of all responses. The frequency of inappropriate responses made in each of the response holes during periods of time-out following premature or incorrect responses was also recorded (Time-out).

The data were subjected to analysis of variance, with surgery as a factor in the analyses. Transformations were applied to reaction time and percentage data (log 10 and angular, respectively) to normalize the distributions in accordance with the ANOVA model (Winer, 1971).

\section{Results}

\section{Histological results}

Figure 2 shows the extent of the cell loss and gliosis. There was no significant difference in lesion size between the groups $[F(1,20)$ $=2.97$, n.s.], although the 3 largest lesions were in the lateral group. For the medial group, mean lesion volume was $5.48 \mathrm{~mm}^{3}$ $(\mathrm{SD}=1.8$; median $=5.4)$ and for the lateral group, $7.75 \mathrm{~mm}^{3}$ $(\mathrm{SD}=5.3 ;$ median $=5.6)$. 
Figure 2. Coronal sections, based on Pellegrino et al. (1979), showing the extent and location of the medial $(l e f t)$ and the lateral (right) striatal lesions, defined by areas of neuronal loss. The black area shows the extent of the damage common to all rats $(n=13$ and 11$)$, shading shows the extent of damage common to 6-10 rats, and stippling shows the extent of damage sustained by $1-5$ rats.

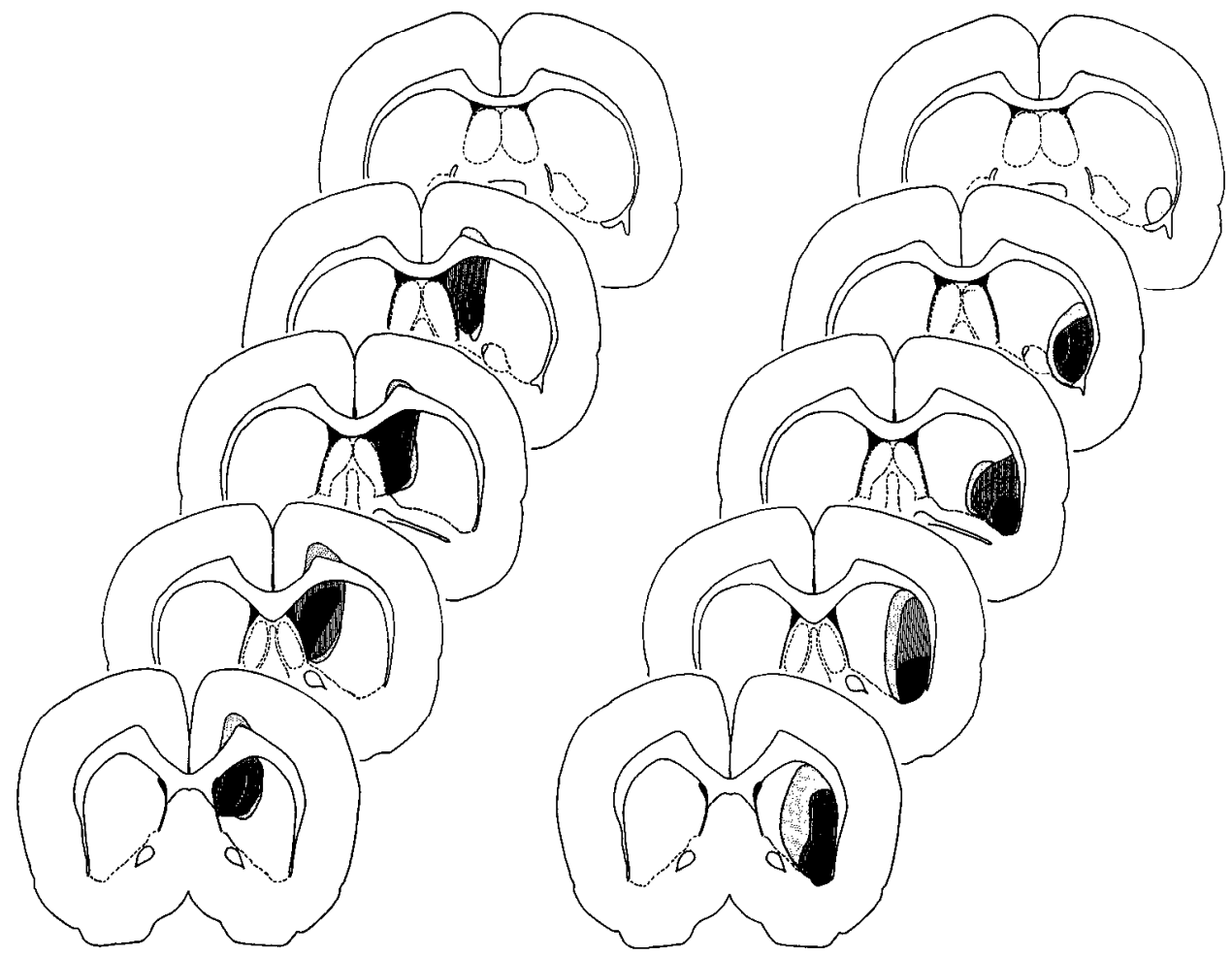

There was some damage to the lower layers of cortical areas Fr1 and Fr2 (frontal cortex) (Zilles, 1985) in a minority $(n=4)$ of the rats with medial lesions.

\section{IT of responses}

The IT for responses in Discrimination 1 was faster than that for responses in Discrimination $2[F(1,21)=7.78, p<0.01]$; the mean IT for each Discrimination was 0.29 and $0.35 \mathrm{sec}$, respectively. There was no interaction between Discrimination and Surgery $[F(1,12)=0.167$, n.s.]; therefore, the IT data for the Discrimination conditions were combined.

Figure 3 shows IT of responses made ipsilateral or contralateral to the side of the lesion, pre- and postoperatively (in the case of preoperative data, ipsi- and contralateral refer to the side of the subsequent lesion). When an animal showed an asymmetry in IT preoperatively, it was lesioned contralateral to the side of the faster times; for this reason, mean contralateral IT was faster than ipsilateral IT $(0.26 \mathrm{vs} 0.36 \mathrm{sec})$.

Postoperatively, the medial lesion group showed an increase in mean IT of contralateral responses to $0.33 \mathrm{sec}$, with the mean IT of ipsilateral responses also $0.33 \mathrm{sec}$ and not significantly different from preoperative values. The lateral lesion group showed no significant change in IT of either ipsi- or contralateral responses, retaining their preoperative IT asymmetry with a mean contralateral IT of $0.27 \mathrm{sec}$ and ipsilateral IT of $0.39 \mathrm{sec}$. This was reflected in a 3-way interaction between the factors of Lesion group, Side of response, and Surgery $[F(1,12)=12.7, p$ $<0.004$ ] and confirmed in the analyses of the simple interactions of Surgery and Side, which was significant for the medial group $[F(1,9)=16.4, p<0.003]$ and not for the lateral group $[F(1,9)=1.04$, n.s. $]$.

There was no significant interaction with delay $[F(3,39)=$ 1.11 , n.s.], indicating that contralateral initiation times were increased in the medial group equally at all delays. The IT effect was also irrespective of the side of stimulus presentation, as there was no significant difference between the Discrimination conditions $[F(1,9)=1.06$, n.s. $]$.

\section{Bias}

Preoperatively, the overall mean percent correct was $89 \%$. The rats were unbiased in their performance of the task, making an equal number of responses to each side in both Discrimination conditions. Postoperatively, percent correct fell to $73 \%$. This was not the result of a general decline in accuracy but rather due to an ipsilateral response bias, reflected in the analysis of percent correct data as an interaction between the factors of Surgery and Side of response $[F(1,20)=29.96, p<0.001]$, with $95.5 \%$ of ipsilateral responses correct but only $50.5 \%$ contralateral responses correct.

Figure 4 shows the postoperative ipsilateral bias of each lesion group, in each of Discrimination 1 and 2. All groups showed some degree of response bias, although the impairment was greater in Discrimination 2-trained animals $[F(1,21)=15.2, p$ $<0.001]$ and also greater in the lateral lesion group $[F(1,21)=$ $7.95, p<0.01]$. There was no significant 3-way interaction between the factors of Lesion, Discrimination, and Surgery $[F(1,21)=0.29$, n.s. $]$, indicating that the postoperative effects of Discrimination and Lesion group were independent and additive.

\section{Time-out responses}

There was a general increase in time-out responding from a mean of 32.4 responses preoperatively to 57.8 responses postoperatively $[F(1,21)=13.4, p<0.001]$. This is a reflection of the greater number of periods of time-out, which resulted from the increase in incorrect responses due to the marked side bias. The increase in frequency of time-out responding was greater in Discrimination 2 than in Discrimination $1[F(1,21)=7.41$, 


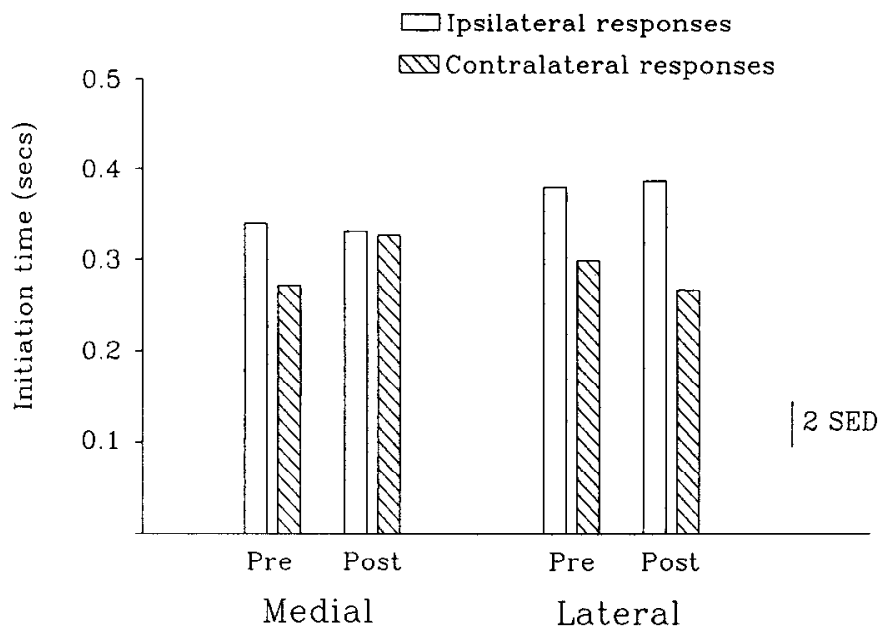

Figure 3. Effect of the lesions on the IT of ipsi- and contralateral responses, pre- and postoperatively (in the case of preoperative data, ipsi- and contralateral refer to the side of the subsequent lesion). The vertical bar indicates 2 SED, representing 2 times the standard error of the difference of the mean values for the interaction between the factors of Lesion site, Surgery, and Side.

$p<0.01$ ], as would be expected from the greater bias in this condition. The increase in the number of periods of time-out was not due to premature withdrawals, as these were not significantly increased postoperatively $[F(1,21)=0.48$, n.s.].

Figure 5 shows the frequency of responses to each side for each lesion group. While the lateral lesion group showed a greater increase in responses to the ipsilateral side, the medial group showed a greater number to the contralateral side. This was reflected in a Lesion group $\times$ Side of response $\times$ Surgery interaction $[F(1,21)=6.55, p<0.02]$. Analysis of the simple interactions revealed the relatively greater increase in ipsilateral responses in the lateral lesion group to be significant $[F(1,22)=$ $6.27, p<0.05$ ], while the greater contralateral responding in the medial lesion group marginally failed to reach significance $[F(1,22)=3.83, p=0.055]$.

\section{Effects of removing the visual discriminanda}

Initiation time. When one of the stimulus lights was removed, there was an increase in the IT of responses to the side normally cued by the missing stimulus. When the ipsilateral stimulus was removed, mean IT of responses normally cued by this stimulus was increased to $0.72 \mathrm{sec}$, while mean IT of contralateral responses within the same session was $0.25 \mathrm{sec}$. Removing the contralateral light, as expected, produced the opposite pattern, with mean IT of responses being $0.71 \mathrm{sec}$ when no stimulus was seen and $0.32 \mathrm{sec}$ to the ipsilateral stimulus.

This pattern was reflected in a Condition $\times$ Side of response interaction $[F(1,26)=430.8, p<0.001]$. There was no interaction with Surgery $[F(1,19)=0.01$, n.s.], showing that the lengthening of initiation times when the discriminanda were removed was still present postoperatively and therefore that the animals continued to attend to both stimuli postoperatively while mediating task performance.

Percent correct. Responding was less accurate when one of the cuing stimuli was removed, with only $57.3 \%$ correct to the missing stimulus compared with $77.3 \%$ correct to the remaining stimulus $[F(1,20)=47.48, p<0.001]$; while the rats always

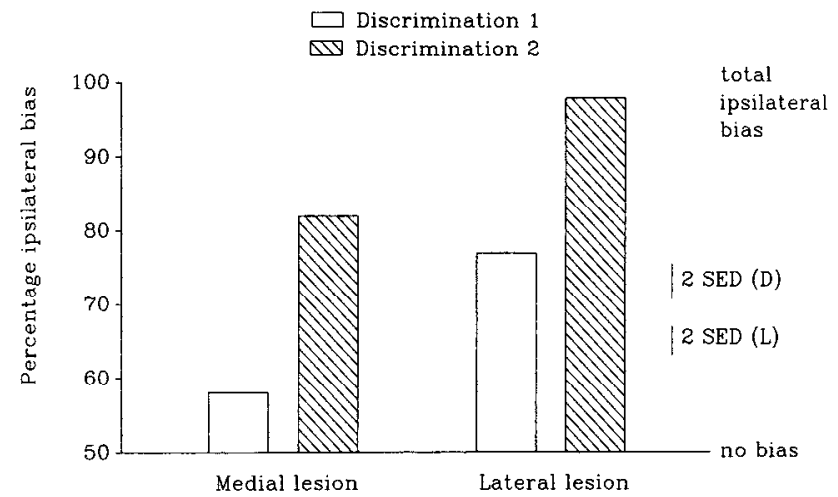

Figure 4. Effect of regional ibotenic acid damage on ipsilateral response bias for the 2 Discrimination conditions. The vertical bars indicate 2 SEDs, representing 2 times the standard error of the difference of the mean values for the main effects of Lesion $(L)$ and Discrimination $(D)$.

made a response, in the absence of the cue light, a response to either side was equally likely.

The effect of surgery was to increase the bias to the ipsilateral side, which resulted in a decrease in contralateral percent correct and an increase in ipsilateral percent correct $[F(1,20)=70.03$, $p<0.001$ ], regardless of the side of the stimulus (Discrimination) or the condition (ipsilateral or contralateral cue removed). Once again, the lateral lesion group was more impaired postoperatively than the medial lesion group $[F(1,20)=5.97, p<$ $0.02]$, but all these effects were additive with the effect of removing the cues, and the 3 -way interaction was not significant $[F(1,18)=1.38$, n.s. $]$.

\section{Effects of presenting both stimuli ("extinction")}

As evident in Figure 6, presenting both stimuli to the rats simultaneously did not enhance the ipsilateral bias seen in the baseline condition $[F(1,20)=0.16$, n.s.]. Bias was greater both in Discrimination 2 postoperatively $[F(1,20)=16.45, p<0.001]$ and in the laterally lesioned animals $[F(1,20)=11.55, p<$ $0.003]$, but these factors did not interact with extinction $[F(1,20)$ $=1.48, \mathrm{n} . \mathrm{s}$. . The similarity of performance in these "extinction" trials to performance in the baseline condition is emphasized.

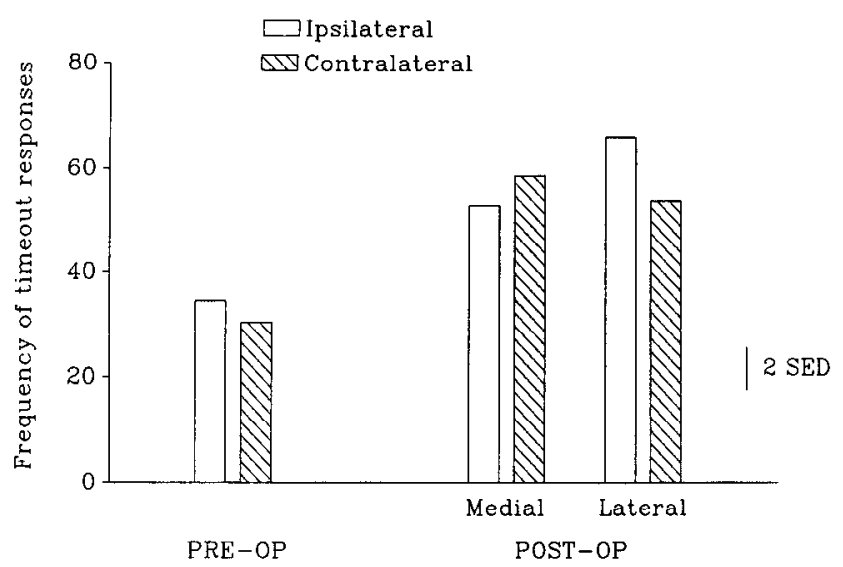

Figure 5. The frequency of inappropriate (time-out) responses made to the ipsi- and contralateral side preoperatively, and by each lesion group postoperatively. Two times the standard error of the difference between the mean values for the interaction of Lesion site, Surgery, and Side, is shown by the vertical bar. 


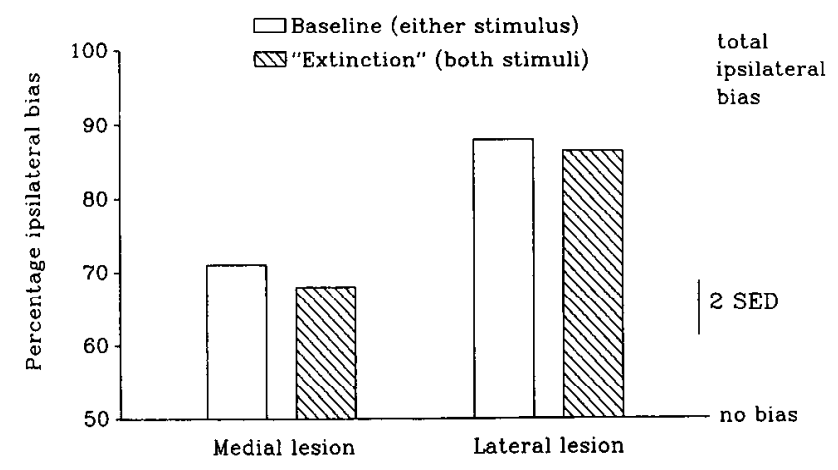

Figure 6. Presenting both stimuli simultaneously did not change the degree of spatial bias compared with the baseline condition. The vertical bar shows 2 times the standard error of the difference between the mean values for the main effect of lesion.

\section{Discussion}

The paradigm used here enabled the dissociation of sensory from response-related behavioral processes and the assessment and quantification of any general, bilateral, effects as well as hemispatial deficits. In accordance with previous reports (Carli et al., 1985, 1989; Mittleman et al., 1988; Brown and Robbins, 1989), these results demonstrate that striatal manipulations result in contralateral response-related impairments and do not disrupt sensory processes; the animals were shown to be attending to both ipsi- and contralateral stimuli postoperatively. Moreover, the results reported here have shown that the roles of the medial and lateral portions of the striatum can be dissociated in the manifestation of neglect, and by using a task which enabled the analysis of different components of behavior, the nature of the impairments following each of the focal lesions was clarified.

Concentrations of ibotenic acid equivalent to those used in the present study have been shown to produce neuronal loss within the caudate putamen of the rat, while sparing oligodendrocytes and fibers of passage (Schwarcz et al., 1979; Coffey et al., 1988). Therefore, the discussion focuses on the nature of the behavioral impairment which is assumed to arise specifically from striatal cell death and not from disruption of corticofugal fibers transversing the caudate putamen.

The demonstration that initiation time was affected only by the medial lesions, whereas spatial response bias was greater following the lateral lesion, has important neuropsychological implications. First, it suggests that these 2 measures may represent different aspects of elementary processes in response selection that are mediated by segregated "functional loops." Second, the retarded contralateral initiation time and ipsilateral response bias reported to follow dopamine depletion of the entire caudate putamen (Carli et al., 1985, 1989) can now be seen to have resulted from disruption of these distinct processes, rather than reflecting aspects of a common psychological deficit.

Detailed comparative analysis of the 2 Discrimination conditions used here enables further specification of the component processes of behavioral neglect. Thus, the common effects of the striatal lesions on initiation time and bias in both conditions indicate that the impairments are response related, rather than resulting from deficits in sensory attention (Carli et al., 1985, 1989). Consistent differences in preoperative performance of the 2 Discriminations can also be used to make inferences about the nature of the underlying psychological processes. The extent of involvement of the striatum in these processes then depends on whether the preoperative differences are preserved, attenuated, or even enhanced postoperatively.

\section{Specification of task-related processes}

Discrimination 2 (responding away from the stimuli) was more difficult than Discrimination 1 (responding towards the side of stimulation), the rats taking longer to learn the task and performing with slower ITs. This is consistent with the human literature on stimulus-response (S-R) compatibility effects (Simon, 1969; Posner et al., 1973) and probably reflects an extra processing load in the selection of the response. Thus, Frith and Done (1986) have postulated that S-R compatibility effects reflect the operation of different "routes to action." For tasks with high S-R compatibility, a "direct" route is used, in which the external stimuli themselves determine the direction of action. For tasks with low compatibility, a matrix of S-R contingencies must first be consulted, via a "slow" route which necessarily lengthens reaction times. Effective use of the S-R matrix must depend upon the integrity of the representation of responses in space, to which appropriate discriminative stimuli must be referred and also on the efficient inhibition of prepotent (i.e., highly compatible) but inappropriate responses. The operation of the S-R matrix not only involves the computation and selection of responses, but is also subject to such factors as changes in response set. We define response set here as the prior assignment of probability of selection from the repertoire of available responses. From this definition, it follows that spatial response bias reflects a disrupted response set (Carli et al., 1989). This disruption has becn shown to result from a distortion in responsc space which leads to a restriction in the available response repertoire (Brown and Robbins, 1989).

\section{Effects of unilateral striatal lesions}

In the present study, the effects of striatal lesions on measures of accuracy and IT were only additive with the effect of S-R compatibility; that is to say, the difference between Discrimination 1 and 2 was preserved. This suggests strongly that the striatum does not contribute to the variations in processing load which lead to differences in reaction time as a function of S-R compatibility. However, spatial response bias was significantly greater in Discrimination 2, which suggests that some other aspect of response selection was affected. This result is important for several reasons. First, the enhanced ipsilateral response bias does not merely result from a stereotyped tendency, either produced by the lesion, or as a strategy for coping with an insoluble task. Further evidence for this is that the difference in bias was also found when both stimuli were presented simultaneously in a test analogous to the clinical test of "extinction" (see Fig. 6). Second, the greater bias in Discrimination 2 shows that one result of being trained to use the "slow" route (see above) is an enhanced behavioral susceptibility to changes in response set. This enhanced behavioral susceptibility is especially evident in the extinction test, when exactly the same stimulus configurations were presented to each group but produced different degrees of response bias.

While the effect of a lesion to either striatal region was to increase ipsilateral response bias, there were differences between the medial and the lateral lesion groups. The medial lesion group was significantly less biased than the lateral lesion for both Discrimination 1 and 2 . The medially lesioned rats, even though 
showing apparently less "neglect," were also impaired in initiating contralateral responses. This lengthening of initiation times for contralateral responses occurred regardless of the Discrimination and could therefore be seen to be due to response related and not sensory processes.

The 2 lesion groups were also dissociated by their patterns of responding in time-out periods. The lateral lesion group responded more to the ipsilateral side, reflecting the bias in the performance of the task. The medial lesion group showed a nearsignificant tendency to be biased, but to the contralateral side. A similar, paradoxical contralateral bias in time-out responding has also been observed in other studies, following either unilateral dopamine depletion from the ventral striatum (Carli et al., 1989) or large unilateral ibotenic acid-induced lesions of the striatum (Mittleman et al., 1988). The results of the present experiment suggest that the medial striatum may contribute to these effects.

The paradoxical contralateral bias in time-out responding is important because (1) it demonstrates once more the independence of lateralized reaction time from spatial response tendencies; initiation times of contralateral responses to the visual stimuli were slower in the medial group; (2) it suggests the disruption of the operation of an independent process which normally inhibits inappropriate responses; and (3) the combination of retarded reaction time to an external (i.e., visual) event and impaired inhibition of inappropriate responses suggests a weakening of attentional control over actions that might normally be attributed to frontal lobe function (Shallice, 1982; Verfaellie and Heilman, 1987).

The present results demonstrate a double dissociation of behavioral changes produced by damage to discrete regions of the striatum. Lesions of the lateral striatum resulted in changes in response bias which reflect changes in response set, which are also seen following medial striatal lesions. However, medial striatal lesions also resulted in a pattern of effects not seen following lateral striatal lesions (lengthened contralateral IT and increased contralateral time-out responses), suggestive of disrupted attentional control over response output. Previous results showed that changes in response set and attention to action occurred together following dopaminc depletion from the entirc striatum, unilaterally. From the present results, it can be seen that the apparent unitary nature of contralateral neglect arises from the disruption of separate functional loops within the striatum, controlling different aspects of response output.

\section{References}

Alexander, G. E., M. R. De Long, and P. L. Strick (1986) Parallel organization of functionally segregated circuits linking basal ganglia and cortex. Annu. Rev. Neurosci. 9: 357-381.

Barth, T. M., S. M. Parker, and H. M. Sinnamon (1982) Unilateral lesions of the anteromedial cortex in the rat impair approach to contralateral visual cues. Physiol. Behav. 29: 141-147.

Benton, A. L. (1956) Jaques Loeb and the method of double stimulation. J. Hist. Med. Allied Sci. 11: 47-53.

Brown, V. I., and T. W. Robbins (1989) Deficits in response space following unilateral ibotenic acid lesions of the striatum. J. Neurosci. 9: 981-987.

Carli, M., T. W. Robbins, J. L. Evenden, and B. J. Everitt (1983) Effects of lesions to ascending noradrenergic neurons on performance of a five choice serial reaction task in rats: Implications for theories of dorsal noradrenergic function based on selective attention and arousal. Behav. Brain Res. 35: 443-457.

Carli, M., J. L. Evenden, and T. W. Robbins (1985) Depletion of unilateral striatal dopamine impairs initiation of contralateral actions and not sensory attention. Nature 313: 679-682.
Carli, M., G. H. Jones, and T. W. Robbins (1989) Effects of unilateral dorsal and ventral striatal dopamine on visual neglect in the rat: A neural and behavioural analysis. Neuroscience 29: 309-327.

Castro, A. J. (1972) The effects of cortical ablations on digital usage in the rat. Brain Res. 37: 173-185.

Coffey, P. J., V. H. Perry, Y. Allen, J. Sinden, and J. N. P. Rawlins (1988) lbotenic acid induced demyelination in the central nervous system: A consequence of a local inflammatory response. Neurosci. Lett. 84: 178-184.

Cowey, A., and T. Bozek (1974) Contralateral "neglect" after unilateral dorsomedial prefrontal lesions in rats. Brain Res. 72: 53-63.

Crowne, D. P., and M. N. Pathria (1982) Some attentional effects of unilateral frontal lesions in the rat. Behav. Brain Res. 6: 25-39.

Divac, I. (1972) Neostriatum and functions of prefrontal cortex. Acta Neurobiol. Exp. 32: 461-477.

Divac, I. (1983) Two levels of functional heterogeneity of the neostriatum. Neuroscience 10:1151-1155.

Divac, I., H. E. Rosvold, and M. K. Szwarcbart (1967) Behavioural effects of selective ablation of the caudate nucleus. J. Comp. Physiol. Psychol. 63: 184-190.

Dunnett, S. B., and S. D. Iversen (1980) Regulatory impairments following selective kainic acid lesions of the neostriatum. Behav. Brain Res. 1: 497-506.

Dunnett, S. B., and S. D. Iversen (1982a) Sensorimotor impairments following localized kainic acid and 6-OHDA lesions of the neostriatum. Brain Res. 248: 121-127.

Dunnett, S. B., and S. D. Iversen (1982b) Regulatory impairments following selective 6-OHDA lesions of the neostriatum. Behav. Brain Res. 4: 195-202.

Dunnett, S. B., A. Björkland, U. Stenevi, and S. D. Iversen (1981) Behavioural recovery following transplantation of substantia nigra in rats subjected to 6-OHDA lesions of the nigrostriatal pathway: I. Unilateral lesions. Brain Res. 215: 147-161.

Fairley, P. C., and J. F. Marshall (1986) Dopamine in the lateral caudate-putamen of the rat is essential for somatosensory orientation. Behav. Neurosci. 100: 652-663.

Frith, C. D., and D. J. Done (1986) Routes to action in reaction time tasks. Psychol. Res. 48: 169-177.

Graybiel, A. M., and C. W. Ragsdale (1983) Biochemical anatomy of the striatum. In Chemical Neuroanatomy, P. C. Emson, ed., pp. 427504, Raven, New York.

Koelle, G. B. (1954) The histochemical localization of cholinesterase in the central nervous system of the rat. J. Comp. Neurol. 100: 211228.

Mittleman, G., V. J. Brown, and T. W. Robbins (1988) Intentional neglect following unilateral ibotenic acid lesions of the striatum. Neurosci. Res. Commun. 2: 1-8.

Nauta, W. J. H., and V. B. Domesick (1984) Afferent and efferent relationships of the basal ganglia. Ciba Found. Symp. 107: 3-23.

Pellegrino, L. J., A. S. Pellegrino, and A. J. Cushman (1979) A Stereotaxic Atlas of the Rat Brain, 2nd ed., Plenum, New York.

Pisa, M. (1988) Motor somatotopy in the striatum of rat: Manipulation, biting and gait. Behav. Brain Res. 27: 21-35.

Posner, M. I., R. M. Klein, J. Summers, and S. Buggie (1973) On the selection of signals. Mem. Cognit. 1: 2-12.

Sabol, K. E., D. B. Neill, S. A. Wages, W. Church, and J. B. Justice (1985) Dopamine depletion in a striatal subregion disrupts performance of a skilled motor task in the rat. Brain Res. 335: 33-43.

Schwarcz, R., T. Hökfelt, K. Fuxe, G. Jonsson, M. Goldstein, and L. Terenius (1979) Ibotenic acid-induced neuronal degeneration: A morphological and neurochemical study. Exp. Brain Res. 37: 199216.

Shallice, T. (1982) Specific impairments of planning. Phil. Trans. R. Soc. London [Biol.] 298: 199-209.

Simon, J. R. (1969) Reactions toward the source of stimulation. J. Exp. Psychol. 81: 174-176.

Verfaellie, M., and K. M. Heilman (1987) Response preparation and response inhibition after lesions of the medial frontal lobe. Arch. Neurol. 44: 1265-1271.

Winer, B. J. (1971) Statistical Principles in Experimental Design, 2nd ed., McGraw-Hill Kogakusha, Tokyo.

Zilles, K. (1985) The Cortex of the Rat, Springer-Verlag, Berlin. 